(c) 2011 IEEE. Personal use of this material is permitted. Permission from IEEE must be obtained for all other uses, in any current or future media, including reprinting/republishing this material for advertising or promotional purposes, creating new collective works, for resale or redistribution to servers or lists, or reuse of any copyrighted component of this work in other works. 


\title{
On Uplink-Downlink Duality of Multi-Hop MIMO Relay Channel
}

\author{
Yue Rong, Senior Member, IEEE, and Muhammad R. A. Khandaker, Student Member, IEEE
}

\begin{abstract}
For two-hop amplify-and-forward (AF) multipleinput multiple-output (MIMO) relay systems, the uplinkdownlink duality has been recently investigated. In this paper, we establish the duality between uplink and downlink multihop AF-MIMO relay channels with any number of hops and any number of antennas at each node, which is a further generalization of several previously established results. We show that in the downlink relay system, signal-to-interference-noise ratios (SINRs) identical to those in the uplink relay system, and vice versa, can be achieved by two approaches. First, with the same total network transmission power constraint, one simply applies Hermitian transposed uplink relay amplifying matrices at relay nodes in the downlink system. Second, with transmission power constraint at each node of the relay network, one can use scaled and Hermitian transposed uplink relay amplifying matrices in the downlink system, with scaling factors obtained by switching power constraints at different nodes of the uplink system. As an application of the uplink-downlink duality, we propose an optimal design of the source precoding matrix and relay amplifying matrices for multi-hop MIMO relay system with a dirty paper coding (DPC) transmitter at the source node.
\end{abstract}

Index Terms-Uplink-downlink duality, MIMO relay, amplifyand-forward.

\section{INTRODUCTION}

Relay communication is well known for being a costeffective approach in improving the energy-efficiency of communication system in the case of long source-destination distance. When nodes in the relay network are equipped with multiple antennas, we call such system a multiple-input multiple-output (MIMO) relay system [1]-[3]. MIMO relays are particularly useful in extending the network coverage and improving the link reliability of the network. For a twohop amplify-and-forward (AF) MIMO relay system with a linear minimal mean-squared error (MMSE) receiver used at the destination node, the structure of the optimal source precoding matrix and the optimal relay amplifying matrix has been derived for a broad class of objective functions [3]. In [4], the optimal source and relay matrices of a multihop AF-MIMO relay system with a successive interference cancellation (SIC) receiver [5] employed at the destination node have been developed.

Manuscript received July 29, 2010; revised January 27, 2011; accepted March 29, 2011. The associate editor coordinating the review of this paper and approving it for publication was Dr. Chia-Chin Chong.

Y. Rong (corresponding author) and M. R. A. Khandaker are with the Department of Electrical and Computer Engineering, Curtin University, Bentley, WA 6102, Australia (e-mail: y.rong@curtin.edu.au; m.khandaker@postgrad.curtin.edu.au).

This work was supported under Australian Research Council's Discovery Projects funding scheme (project numbers DP110100736, DP110102076).
Recently, the uplink-downlink duality of two-hop AFMIMO relay systems has been derived in [6]. It is shown that for any relay amplifying matrix used in the uplink channel, duality holds when a scaled Hermitian transpose of this matrix is employed in the downlink channel, where the scaling factor is obtained by switching the transmission power constraints at the source and the relay nodes. This result can be seen as a generalization of the well-known duality result for singlehop MIMO systems [7], [8]. For a multi-hop AF-MIMO relay network with single antenna source and destination nodes, the uplink-downlink duality has been established in [9].

In this paper, we extend the uplink-downlink duality results in [6]-[9] to multi-hop AF-MIMO relay systems with any number of hops and any number of antennas at each node. We define duality as the achievement of identical signalto-interference-noise ratios (SINRs) at the uplink and the downlink systems with the same amount of total network transmission power. The reasons of considering SINR are two folds: First, SINR is an important parameter in communication system in the sense that it directly determines the qualityof-service (QoS) of each data stream. Second, many other parameters such as the achievable data rate and the MSE of signal estimation are closely related to SINR [10]. In particular, we show that for any number of hops, duality can be achieved by two approaches. First, if there is only total network transmission power constraint and no power constraint at individual nodes, then duality holds if $\mathbf{F}_{l}$ and $\mathbf{F}_{L-l}^{H}, l=1, \cdots, L-1$, are used as the relay amplifying matrices at the $l$ th relay node of the downlink and the uplink MIMO relay systems, respectively. Here $(\cdot)^{H}$ denotes matrix Hermitian transpose, and $L$ is the number of hops of the relay network. Second, with transmission power constraint at each node of the relay network, duality can be achieved by applying $c_{l} \mathbf{F}_{l}$ and $\mathbf{F}_{L-l}^{H}$ respectively as the amplifying matrices at the $l$ th relay node of the downlink and the uplink relay systems, $l=1, \cdots, L-1$, where the scaling factor $c_{l}$ is obtained by switching the power constraints at the $l$ th node of the downlink system and the $(L+1-l)$-th node of the uplink system, $l=1, \cdots, L$. For both approaches, the source precoding matrix and the destination receiving matrix in the downlink system are swapped with the destination receiving matrix and the source precoding matrix at the uplink system, respectively.

Furthermore, we prove that the two approaches developed above are not only valid for relay systems with linear transceivers at the source and the destination nodes, but also hold if an SIC-based receiver is used at the destination node of the uplink MIMO relay system, and a transmitter based on dirty paper coding (DPC) [11] is employed at the source node 
of the downlink MIMO relay channel. Interestingly, we show that the two duality approaches can be extended to multiuser AF-MIMO relay systems with any number of multi-antenna users.

As an application of the uplink-downlink duality theorem, we propose an optimal design of the source precoding matrix and relay amplifying matrices for multi-hop AF-MIMO relay systems with a DPC-based transmitter at the source node, by exploiting the results obtained for the dual uplink relay system [4]. Simulation results demonstrate that the optimal DPCbased MIMO relay system has a better bit-error-rate (BER) performance compared with the optimal relay system using the SIC receiver, because the SIC receiver suffers from the error propagation effect, while the DPC transmitter does not.

The rest of this paper is organized as follows. In Section II, we introduce the model of uplink and downlink multi-hop AFMIMO relay communication systems. The duality theorems are proven in Section III. An optimal multi-hop MIMO relay system with a DPC-based transmitter at the source node is developed in Section IV. In Section V, we show some numerical examples. Conclusions are drawn in Section VI.

\section{SySTEM MODEL}

We consider a wireless communication system with one source node, one destination node, and $L-1(L \geq 2)$ relay nodes. We assume that due to the propagation path-loss, the signal transmitted by the $l$ th node can only be received by its direct forward node, i.e., the $(l+1)$-th node. Thus, signals transmitted by the source node pass through $L$ hops until they reach the destination node. We also assume that the number of antennas at each node is $N_{l}, l=1, \cdots, L+1$, and the number of source symbols in each transmission is $N_{b}$. A linear nonregenerative relay matrix is used at each relay node to amplify and forward the received signals. The system block diagrams of downlink and uplink multi-hop AF-MIMO relay systems are shown in Fig. 1.

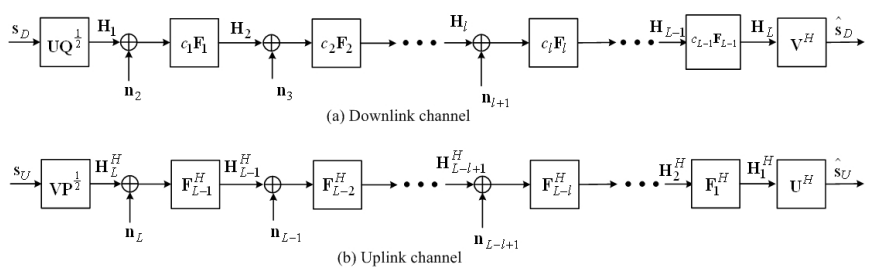

Fig. 1. Block diagrams of downlink and uplink multi-hop AF-MIMO relay systems.

We would like to mention that for AF-MIMO relay systems with linear transceivers at the source and destination nodes, there should be $N_{b} \leq \min \left(N_{1}, N_{2}, \cdots, N_{L+1}\right)$ in order to support $N_{b}$ data streams in one transmission. However, if a nonlinear transmitter is installed at the source node or a nonlinear receiver is installed at the destination node of a MIMO relay system, $N_{b}$ can be greater than $\min \left(N_{1}, N_{2}, \cdots, N_{L+1}\right)$ [4].

In the case of downlink communication, the $N_{b} \times 1$ source symbol vector $\mathbf{s}_{D}=\left[s_{1}, s_{2}, \cdots, s_{N_{b}}\right]^{T}$ at the source node is linearly precoded by the $N_{1} \times N_{b}$ matrix $\mathbf{U Q}^{\frac{1}{2}}$, where
$\mathbf{Q}=\operatorname{diag}\left(q_{1}, q_{2}, \cdots, q_{N_{b}}\right)$ and $\mathbf{U}=\left[\mathbf{u}_{1}, \mathbf{u}_{2}, \cdots, \mathbf{u}_{N_{b}}\right]$ with $\left\|\mathbf{u}_{i}\right\|_{2}=1, i=1, \cdots, N_{b}$. Here $(\cdot)^{T}$ stands for matrix (vector) transpose, $\operatorname{diag}(\cdot)$ denotes a diagonal matrix, $\|\cdot\|_{2}$ stands for the vector Euclidean norm, and $q_{i}, i=1, \cdots, N_{b}$, is the power assigned to the $i$ th data stream. We assume that $\mathrm{E}\left[\mathbf{s}_{D} \mathbf{s}_{D}^{H}\right]=\mathbf{I}_{N_{b}}$, where $\mathrm{E}[\cdot]$ stands for the statistical expectation, and $\mathbf{I}_{n}$ is an $n \times n$ identity matrix. The $N_{1} \times 1$ linearly precoded symbol vector $\mathbf{x}_{1}^{D}=\mathbf{U Q}^{\frac{1}{2}} \mathbf{s}_{D}$ is transmitted by the source node. The $N_{l} \times 1$ signal vector received at the $l$ th node is written as

$$
\mathbf{y}_{l}^{D}=\mathbf{H}_{l-1} \mathbf{x}_{l-1}^{D}+\mathbf{n}_{l}, \quad l=2, \cdots, L+1
$$

where $\mathbf{H}_{l}, l=1, \cdots, L$, is the $N_{l+1} \times N_{l}$ MIMO channel matrix between the $(l+1)$-th and the $l$ th node, i.e., the $l$ th hop, $\mathbf{n}_{l}$ is the $N_{l} \times 1$ independent and identically distributed (i.i.d.) additive white Gaussian noise (AWGN) vector at the $l$ th node, and $\mathbf{x}_{l-1}^{D}$ is the $N_{l-1} \times 1$ signal vector transmitted by the $(l-1)$-th node. We assume that all noises are complex circularly symmetric with zero mean and unit variance.

Using the AF scheme, the input-output relationship at node $l$ is given by

$$
\mathbf{x}_{l}^{D}=c_{l-1} \mathbf{F}_{l-1} \mathbf{y}_{l}^{D}, \quad l=2, \cdots, L
$$

where $c_{l-1} \mathbf{F}_{l-1}$ is the $N_{l} \times N_{l}$ amplifying matrix at node $l$ (the $(l-1)$-th relay node), $c_{l}>0$ is a scaling coefficient which is important for studying the uplink-downlink duality [6] and will be explained later. Combining (1) and (2), the received signal vector at the $l$ th node of the downlink MIMO relay channel is given by

$$
\begin{aligned}
\mathbf{y}_{2}^{D}= & \mathbf{H}_{1} \mathbf{U} \mathbf{Q}^{\frac{1}{2}} \mathbf{s}_{D}+\mathbf{n}_{2} \\
\mathbf{y}_{l+1}^{D}= & \mathbf{H}_{l} \bigotimes_{m=l-1}^{1}\left(c_{m} \mathbf{F}_{m} \mathbf{H}_{m}\right) \mathbf{U} \mathbf{Q}^{\frac{1}{2}} \mathbf{s}_{D}+\mathbf{n}_{l+1} \\
& +\sum_{k=2}^{l} \bigotimes_{m=l}^{k}\left(c_{m-1} \mathbf{H}_{m} \mathbf{F}_{m-1}\right) \mathbf{n}_{k}, \quad l=2, \cdots, L
\end{aligned}
$$

where for matrices $\mathbf{A}_{i}, \bigotimes_{i=l}^{k}\left(\mathbf{A}_{i}\right) \triangleq \mathbf{A}_{l} \cdots \mathbf{A}_{k}$. At the destination node, a linear receiver with an $N_{L+1} \times N_{b}$ weight matrix $\mathbf{V}$ is used to estimate the source symbol vector $\mathbf{s}_{D}$. The estimated symbol vector $\hat{\mathbf{s}}_{D}$ is given by

$$
\hat{\mathbf{s}}_{D}=\mathbf{V}^{H} \mathbf{y}_{L+1}^{D} \text {. }
$$

Since scaling the columns of $\mathbf{V}$ does not change the SINRs at the destination node, we assume that $\left\|\mathbf{v}_{i}\right\|_{2}=1, i=$ $1, \cdots, N_{b}$.

For the uplink MIMO relay system, the communication direction is reversed, and the roles of the source node and the destination node are swapped. The channel matrices are replaced by the Hermitian transpose of channel matrices in the downlink channel. Now the source node applies $\mathbf{V P}^{\frac{1}{2}}$ to precode the uplink source symbol vector $\mathbf{s}_{U}$, where $\mathbf{P}=$ $\operatorname{diag}\left(p_{1}, p_{2}, \cdots, p_{N_{b}}\right)$, and $p_{i}, i=1, \cdots, N_{b}$, is the power assigned to the $i$ th data stream. The $l$ th node, $l=2, \cdots, L$, uses $\mathbf{F}_{L+1-l}^{H}$ to amplify and forward received signals. Similar to (3) and (4), the received signal vector at the $l$ th node of the 
uplink MIMO relay system can be written as

$$
\begin{aligned}
\mathbf{y}_{2}^{U}= & \mathbf{H}_{L}^{H} \mathbf{V} \mathbf{P}^{\frac{1}{2}} \mathbf{s}_{U}+\mathbf{n}_{L} \\
\mathbf{y}_{L+2-l}^{U}= & \bigotimes_{m=l}\left(\mathbf{H}_{m}^{H} \mathbf{F}_{m}^{H}\right) \mathbf{H}_{L}^{H} \mathbf{V} \mathbf{P}^{\frac{1}{2}} \mathbf{s}_{U}+\mathbf{n}_{l} \\
& +\sum_{k=l}^{L-1} \bigotimes_{m=l}^{k}\left(\mathbf{H}_{m}^{H} \mathbf{F}_{m}^{H}\right) \mathbf{n}_{k+1}, \quad l=1, \cdots, L-1 .
\end{aligned}
$$

Finally, the destination node applies $\mathbf{U}$ to estimate the transmitted symbol vector with

$$
\hat{\mathbf{s}}_{U}=\mathbf{U}^{H} \mathbf{y}_{L+1}^{U} .
$$

We would like to note that at this point, for both the downlink and uplink systems, there is no specific design for $\mathbf{U}, \mathbf{V}$, and $\mathbf{F}_{l}, l=1, \cdots, L-1$.

In this paper, the channel state information (CSI) requirement is the same as that in [4]. Basically, we assume that the source node has the CSI knowledge of the first-hop channel, the destination node knows the receiver weight matrix and each relay node knows the CSI of its backward channel and its forward channel. In practice, the backward CSI can be obtained through standard training methods. The forward CSI required at one relay node is exactly the backward CSI at its direct forward relay node, and thus can be obtained by a feedback from its direct forward relay node.

\section{UPLINK-DownLINK DUALITY}

In this section, we investigate the duality between the uplink and the downlink multi-hop AF-MIMO relay systems with any number of hops and any number of antennas at each node. It can be seen from Section II that given an uplink MIMO relay system, constructing its dual downlink MIMO relay system boils down to determining the appropriate relay scaling factors $c_{l}, l=1, \cdots, L-1$, and the source power loading matrices $\mathbf{Q}$. The following two theorems establish the uplink-downlink duality property of multi-hop MIMO relay communication system with any number of hops.

THEOREM 1: If linear transceivers are used at the source and destination nodes of the uplink and the downlink systems, and there is no specific transmission power constraint at each node, then for any $L \geq 2$, the uplink-downlink duality can be achieved by $c_{l}=1, l=1, \cdots, L-1$. With transmission power constraint at individual nodes, duality is attained by setting $\rho_{L+1-l}^{U}=\rho_{l}^{D}, l=1, \cdots, L$, and $c_{l}, l=1, \cdots, L-1$, are obtained by transmission power constraints. Here $\rho_{l}^{U} \geq 0$ and $\rho_{l}^{D} \geq 0, l=1, \cdots, L$, are the power budgets at the $l$ th node of the uplink and the downlink systems, respectively.

Proof: See Appendix A.

It can be seen from Theorem 1 that if there is only total network transmission power constraint and no power constraint at individual nodes, then duality holds if $\mathbf{F}_{l}$ and $\mathbf{F}_{L-l}^{H}, l=1, \cdots, L-1$, are used as the amplifying matrix at the $l$ th relay node of the downlink and the uplink MIMO relay systems, respectively. However, in some practical applications, there is transmission power constraint at each node of the relay network. In such case, as suggested by Theorem 1, duality can be achieved by applying $c_{l} \mathbf{F}_{l}$ and $\mathbf{F}_{L-l}^{H}$ respectively as the amplifying matrix at the $l$ th relay node of the downlink and the uplink relay systems, $l=1, \cdots, L-1$, where the scaling factor $c_{l}$ is determined by switching the power constraints at the $l$ th node of the downlink system and the $(L+1-l)$-th node of the uplink system, $l=1, \cdots, L$. It is worth noting that Theorem 1 holds for any transceiver matrices $\mathbf{U}, \mathbf{V}$, and relay amplifying matrices $\mathbf{F}_{l}, l=1, \cdots, L-1$. However, for the uplink MIMO relay system, if a linear MMSE receiver is used, the optimal $\mathbf{U}, \mathbf{V}$, and $\mathbf{F}_{l}, l=1, \cdots, L-1$, are derived in [12].

Interestingly, Theorem 1 includes the results in [6]-[9] as special cases. It extends the uplink-downlink duality results from single-hop MIMO systems and two-hop AF-MIMO relay systems to multi-hop AF-MIMO relay systems with any number of hops and any number of antennas at each node.

TheOrem 2: If a DPC-based transmitter is used at the source node of the downlink MIMO relay system, and an SIC-based receiver is employed at the destination node of the uplink MIMO relay system, the uplink-downlink duality can be achieved by $c_{l}=1, l=1, \cdots, L-1$, when there is no specific transmission power constraint at each node. With transmission power constraint at individual nodes, duality can be attained by setting $\rho_{L+1-l}^{U}=\rho_{l}^{D}, l=1, \cdots, L$, and $c_{l}, l=1, \cdots, L-1$, are obtained by transmission power constraints.

ProOf: See Appendix B.

Theorem 2 extends the duality results in Theorem 1 to the scenario where nonlinear transceivers are used at the source node of the downlink channel and the destination node of the uplink channel, respectively. Similar to Theorem 1, Theorem 2 holds for any transceiver matrices $\mathbf{U}, \mathbf{V}$, and relay amplifying matrices $\mathbf{F}_{l}, l=1, \cdots, L-1$. However, if a nonlinear MMSESIC receiver is used at the uplink MIMO relay system, the optimal $\mathbf{U}, \mathbf{V}$, and $\mathbf{F}_{l}, l=1, \cdots, L-1$, can be found in [4]. Interestingly, both Theorem 1 and Theorem 2 also hold for multiuser MIMO relay scenario as explained below. In a broadcast channel (BC), a central station broadcasts information through $L$ hops to $M$ users each having $R_{m}$ antennas, $m=1, \cdots, M$, while in a multiaccess channel (MAC), $M$ users each having $T_{m}$ antennas, $m=1, \cdots, M$, send information to a central station via $L$ hops. The BC channel can be equivalently treated as a downlink multihop MIMO relay channel by grouping all users to form a "super" destination node with $N_{L+1}=\sum_{m=1}^{M} R_{m}$ antennas. Accordingly, one can view the MAC channel as an uplink multi-hop MIMO channel with $N_{1}=\sum_{m=1}^{M} T_{m}$ antennas at the source node. Obviously, duality holds for the $\mathrm{BC}$ and MAC, provided that $\mathbf{V}$ (the destination receiving matrix in the $\mathrm{BC}$ and the source precoding matrix in the MAC) is chosen as a block diagonal matrix.

\section{DPC-Based Optimal Multi-Hop Mimo Relay DESIGN}

In a DPC-based multi-hop MIMO relay system, the source symbol vector $\mathbf{s}_{D}$ is formed by encoding the informationbearing symbols $\mu_{i}, i=1, \cdots, N_{b}$, successively by removing the interference from the symbols already encoded. Thus, 
compared with a MIMO relay system using a linear transmitter at the source node, the DPC-based relay system should have a better BER performance.

The DPC-based optimal multi-hop MIMO relay design problem can be formulated as

$$
\begin{aligned}
\min _{\mathbf{U}, \mathbf{Q},\left\{c_{l} \mathbf{F}_{l}\right\}} & f\left(\mathbf{U}, \mathbf{Q},\left\{c_{l} \mathbf{F}_{l}\right\}\right) \\
\text { s.t. } & P_{l}^{D} \leq \rho_{l}^{D}, \quad l=1, \cdots, L
\end{aligned}
$$

where $f(\cdot)$ stands for a unified objective function [3], and $P_{l}^{D}, l=1, \cdots, L$, is the power consumed by the $l$ th node in the downlink system given by (27)-(29) in Appendix A. Function $f(\cdot)$ includes a broad class of frequently used objective functions in MIMO system design such as the negative source-destination mutual information, and the MSE of the signal waveform estimation at the destination. Directly solving the problem of (9) and (10) is difficult for a unified objective function $f(\cdot)$ and MIMO relay systems with a DPC transmitter, even for a single-hop (point-to-point) MIMO system [10]. Based on our knowledge, the problem of (9) and (10) has not been directly solved in literature. Now we apply Theorem 2 to optimize the DPC-based MIMO relay system. First, the optimization problem for the dual SIC-based uplink MIMO relay system can be written as

$$
\begin{aligned}
\min _{\mathbf{V}, \mathbf{P},\left\{\mathbf{F}_{L-l}^{H}\right\}} & f\left(\mathbf{V}, \mathbf{P},\left\{\mathbf{F}_{L-l}^{H}\right\}\right) \\
\text { s.t. } & P_{l}^{U} \leq \rho_{L+1-l}^{D},
\end{aligned}
$$

where $P_{l}^{U}, l=1, \cdots, L$, is the power consumed by the $l$ th node in the uplink system given by (22)-(24) in Appendix A. Applying the majorization theory [13], the problem of (11) and (12) has been solved in [4] for a broad class of objective functions $f(\cdot)$. The optimal feed-forward matrix $\mathbf{W}$ at the destination node of the uplink MIMO relay system has also been developed in [4]. Note that for a single-hop MIMO system, the uplink-downlink duality has been applied in [10] to optimize the DPC-based transceiver design.

In this section, we take the optimal $\mathbf{V}, \mathbf{P}$, and $\mathbf{F}_{l}, l=$ $1, \cdots, L-1$, from [4]. Based on Theorem 2, the optimal source precoding matrix in the DPC-based downlink relay system can be written as $\mathbf{U Q}^{\frac{1}{2}}$, where $\mathbf{U}=\left[\overline{\mathbf{w}}_{1}, \overline{\mathbf{w}}_{2}, \cdots, \overline{\mathbf{w}}_{N_{b}}\right]$, and $\overline{\mathbf{w}}_{i}, i=1, \cdots, N_{b}$, are obtained by scaling columns of $\mathbf{W}$ such that $\left\|\overline{\mathbf{w}}_{i}\right\|_{2}=1, i=1, \cdots, N_{b}$. The optimal amplifying matrix at the $l$ th relay node of the DPC-based relay system is $c_{l} \mathbf{F}_{l}$, where the scalar $c_{l}$ is determined by the transmission power budget $\rho_{l+1}^{D}$ as explained later. The optimal receiving matrix at the destination node of the DPC-based relay system is $\mathbf{V} \boldsymbol{\Gamma}$, where $\boldsymbol{\Gamma}=\operatorname{diag}\left(\gamma_{1}, \gamma_{2}, \cdots, \gamma_{N_{b}}\right)$ with $\gamma_{i}$ chosen such that $\gamma_{i} \sqrt{q_{i}} \mathbf{v}_{i}^{H} \mathbf{H}_{L} \bigotimes_{m=L-1}^{1}\left(c_{m} \mathbf{F}_{m} \mathbf{H}_{m}\right) \overline{\mathbf{w}}_{i}=1$, $i=1, \cdots, N_{b}$.

Now the task is to obtain the unknown quantities $q_{i}, i=$ $1, \cdots, N_{b}$, and $c_{l}, l=1, \cdots, L-1$. Towards this goal, we assume for the moment that $\boldsymbol{\Gamma}=\mathbf{I}_{N_{b}}$, since scaling the receiving vector $\mathbf{v}_{i}$ does not change $\operatorname{SINR}_{i}^{D}, i=1, \cdots, N_{b}$, in (40) of Appendix B. With a DPC encoder at the source node, the information-bearing symbol $\mu_{i}$ is encoded into $s_{i}$ by treating $\sum_{j=1}^{i-1} \sqrt{q_{j}} \mathbf{v}_{i}^{H} \mathbf{H}_{L} \bigotimes_{m=L-1}^{1}\left(c_{m} \mathbf{F}_{m} \mathbf{H}_{m}\right) \overline{\mathbf{w}}_{j} s_{j}$ as the interference known at the transmitter. Note that the interference term $\sum_{j=i+1}^{N_{b}} \sqrt{q_{j}} \mathbf{v}_{i}^{H} \mathbf{H}_{L} \bigotimes_{m=L-1}^{1}\left(c_{m} \mathbf{F}_{m} \mathbf{H}_{m}\right)$ $\overline{\mathbf{w}}_{j} s_{j}$ is unknown at this stage. At the destination node, after applying the linear filter $\mathbf{V}$ and the DPC decoder, the estimated information-bearing symbol can be written as

$$
\begin{aligned}
& \hat{\mu}_{i}=\sqrt{q_{i}} \mathbf{v}_{i}^{H} \mathbf{H}_{L} \bigotimes_{m=L-1}^{1}\left(c_{m} \mathbf{F}_{m} \mathbf{H}_{m}\right) \overline{\mathbf{w}}_{i} \mu_{i} \\
& +\sum_{j=i+1}^{N_{b}} \sqrt{q_{j}} \mathbf{v}_{i}^{H} \mathbf{H}_{L} \bigotimes_{m=L-1}^{1}\left(c_{m} \mathbf{F}_{m} \mathbf{H}_{m}\right) \overline{\mathbf{w}}_{j} s_{j} \\
& +\mathbf{v}_{i}^{H}\left(\sum_{l=2}^{L} \bigotimes_{m=L}^{l}\left(c_{m-1} \mathbf{H}_{m} \mathbf{F}_{m-1}\right) \mathbf{n}_{l}+\mathbf{n}_{L+1}\right), \quad i=1, \cdots, N_{b}
\end{aligned}
$$

with the output SINR $\beta_{i}$ as

$$
\beta_{i}=\frac{d_{i, i} q_{i}}{\sum_{j=i+1}^{N_{b}} d_{i, j} q_{j}+e_{i}+\mathbf{g}_{i}^{T} \boldsymbol{\theta}}, \quad i=1, \cdots, N_{b}
$$

where $\boldsymbol{\theta}$ is an $(L-1) \times 1$ vector with elements $\theta_{l}=1 / \prod_{m=1}^{l} c_{m}^{2}, l=1, \cdots, L-1, \mathbf{g}_{i}$ is an $(L-$ 1) $\times 1$ vector with elements given by $g_{i, L-1}=1$, $g_{i, l}=\mathbf{v}_{i}^{H} \bigotimes_{m=L}^{l+2}\left(\mathbf{H}_{m} \mathbf{F}_{m-1}\right) \bigotimes_{m=l+2}^{L}\left(\mathbf{F}_{m-1}^{H} \mathbf{H}_{m}^{H}\right) \mathbf{v}_{i}, l=$ $1, \cdots, L-2$, and

$$
\begin{aligned}
d_{i, j} & \triangleq\left|\mathbf{v}_{i}^{H} \mathbf{H}_{L} \bigotimes_{m=L-1}^{1}\left(\mathbf{F}_{m} \mathbf{H}_{m}\right) \overline{\mathbf{w}}_{j}\right|^{2}, \quad j=i, \cdots, N_{b} \\
e_{i} & \triangleq \mathbf{v}_{i}^{H} \bigotimes_{m=L}^{2}\left(\mathbf{H}_{m} \mathbf{F}_{m-1}\right) \bigotimes_{m=2}^{L}\left(\mathbf{F}_{m-1}^{H} \mathbf{H}_{m}^{H}\right) \mathbf{v}_{i} .
\end{aligned}
$$

Collecting all equations in (13) for $i=1, \cdots, N_{b}$, we obtain the following systems of linear equations

$$
\boldsymbol{\Phi} \mathbf{q}+\mathbf{G}^{T} \boldsymbol{\theta}=-\mathbf{e}
$$

where $\mathbf{q} \triangleq\left[q_{1}, q_{2}, \cdots, q_{N_{b}}\right]^{T}, \mathbf{e} \triangleq\left[e_{1}, e_{2}, \cdots, e_{N_{b}}\right]^{T}, \boldsymbol{\Phi}$ is an $N_{b} \times N_{b}$ upper-triangle matrix with elements of $\phi_{i, j}=0, j=$ $1, \cdots, i-1, \phi_{i, i}=-d_{i, i} / \beta_{i}, \phi_{i, j}=d_{i, j}, j=i+1, \cdots, N_{b}$, $i=1, \cdots, N_{b}$, and $\mathbf{G}$ is an $(L-1) \times N_{b}$ matrix whose $i$ th column is given by $\mathbf{g}_{i}$ in (13).

From the transmission power consumed at the relay nodes (27) and (28) in Appendix A, we have

$$
\begin{aligned}
\frac{P_{l+1}^{D}}{\prod_{m=1}^{l} c_{m}^{2}}= & \mathbf{a}_{l}^{T} \mathbf{q}+\xi_{l, 1}+\sum_{k=1}^{l-1} \frac{\xi_{l, k+1}}{\prod_{m=1}^{k} c_{m}^{2}} \\
& l=2, \cdots, L-1 \\
P_{2}^{D} / c_{1}^{2}= & \mathbf{a}_{1}^{T} \mathbf{q}+\xi_{1,1}
\end{aligned}
$$

where $\mathbf{a}_{l}$ is an $N_{b} \times 1$ vector with elements $a_{l, i}=$ $\overline{\mathbf{w}}_{i}^{H} \bigotimes_{m=1}^{l}\left(\mathbf{H}_{m}^{H} \mathbf{F}_{m}^{H}\right) \bigotimes_{m=l}^{1}\left(\mathbf{F}_{m} \mathbf{H}_{m}\right) \overline{\mathbf{w}}_{i}, i=1, \cdots, N_{b}$, and $\xi_{l, k} \triangleq\left\{\begin{array}{cc}\operatorname{tr}\left(\mathbf{F}_{l} \bigotimes_{m=l}^{k+1}\left(\mathbf{H}_{m} \mathbf{F}_{m-1}\right) \bigotimes_{m=k+1}^{l}\left(\mathbf{F}_{m-1}^{H} \mathbf{H}_{m}^{H}\right) \mathbf{F}_{l}^{H}\right), \\ k=1, \cdots, l-1 \\ \operatorname{tr}\left(\mathbf{F}_{l} \mathbf{F}_{l}^{H}\right), & k=l\end{array}\right.$ where $\operatorname{tr}(\cdot)$ stands for matrix trace. Equation (15) can be rewritten as

$$
\begin{array}{r}
\sum_{k=1}^{l-1} \frac{\xi_{l, k+1}}{\prod_{m=1}^{k} c_{m}^{2}}-\frac{P_{l+1}^{D}}{\prod_{m=1}^{l} c_{m}^{2}}=-\mathbf{a}_{l}^{T} \mathbf{q}-\xi_{l, 1} \\
l=2, \cdots, L-1 .
\end{array}
$$


Collecting all equations in (17) for $l=2, \cdots, L-1$, and together with (16), we obtain the following system of equations

$$
\boldsymbol{\Psi} \boldsymbol{\theta}=-\mathbf{A}^{T} \mathbf{q}-\boldsymbol{\xi}
$$

where $\boldsymbol{\Psi}$ is an $(L-1) \times(L-1)$ lower-triangle matrix with elements of $\psi_{l, k}=\xi_{l, k+1}, k=1, \cdots, l-1, \psi_{l, l}=-P_{l+1}^{D}$, $\psi_{l, k}=0, k=l+1, \cdots, L-1, l=1, \cdots, L-1, \mathbf{A}$ is an $N_{b} \times(L-1)$ matrix whose $l$ th column is given by $\mathbf{a}_{l}$ in (16) and (17), and $\boldsymbol{\xi} \triangleq\left[\xi_{1,1}, \xi_{2,1}, \cdots, \xi_{L-1,1}\right]^{T}$.

Now (14) and (18) form a system of linear equations of $\boldsymbol{\theta}$ and q. Solving (14) and (18), we obtain

$$
\begin{aligned}
& \mathbf{q}=\left(\boldsymbol{\Phi}-\mathbf{G}^{T} \boldsymbol{\Psi}^{-1} \mathbf{A}^{T}\right)^{-1}\left(\mathbf{G}^{T} \boldsymbol{\Psi}^{-1} \boldsymbol{\xi}-\mathbf{e}\right) \\
& \boldsymbol{\theta}=-\boldsymbol{\Psi}^{-1}\left[\boldsymbol{\xi}+\mathbf{A}^{T}\left(\boldsymbol{\Phi}-\mathbf{G}^{T} \boldsymbol{\Psi}^{-1} \mathbf{A}^{T}\right)^{-1}\left(\mathbf{G}^{T} \boldsymbol{\Psi}^{-1} \boldsymbol{\xi}-\mathbf{e}\right)\right] .
\end{aligned}
$$

Note that $\boldsymbol{\Psi}^{-1}$ always exists because $\boldsymbol{\Psi}$ is a lower-triangle matrix, and $\boldsymbol{\Phi}-\mathbf{G}^{T} \boldsymbol{\Psi}^{-1} \mathbf{A}^{T}$ is invertible since $\boldsymbol{\Phi}$ is an uppertriangle matrix and $\boldsymbol{\Psi}^{-1}$ is a lower-triangle matrix. Finally, $c_{l}$ can be obtained from (20) with $c_{1}=\sqrt{1 / \theta_{1}}$ and $c_{l}=$ $\sqrt{\theta_{l-1} / \theta_{l}}, l=2, \cdots, L-1$.

\section{NUMERICAL EXAMPLES}

In this section, we study the performance of the proposed DPC-based source precoding matrix and relay amplifying matrices through numerical simulations. To precode the $i$ th information-bearing symbol $\mu_{i}$ into the source symbol $s_{i}, i=$ $1, \cdots, N_{b}$, we apply the Tomlinson-Harashima coding technique [14] and [15], which is a simple but suboptimal implementation of the DPC scheme. The Tomlinson-Harashima scheme makes use of the modulo operation to remove the interference from the preceding symbols without increasing the transmission power at the source node. Accordingly, the length of the modulo $\Delta$ is chosen to preserve the transmission power consistency.

The source, destination, and all relay nodes are equipped with multiple antennas. We simulate a flat Rayleigh fading environment where the channel matrices have i.i.d. entries with zero mean and variances $\sigma_{l}^{2} / N_{l}$ for $\mathbf{H}_{l}, l=1, \cdots, L$. For each channel realization, 1000 QAM-modulated symbols with Gray mapping are transmitted at each data stream, and all simulation results are averaged over 500 independent channel realizations. We define $\mathrm{SNR}_{l} \triangleq \sigma_{l}^{2} \rho_{l}^{D} N_{l+1} / N_{l}$ as the signal-to-noise ratio (SNR) of the $l$ th hop, $l=1, \cdots, L$. The BER performance of the proposed optimal DPC-based relay system is compared with that of the optimal relay system using the SIC receiver [4]. As a benchmark, we also show the performance of the fictitious genie-aided SIC-based relay system, where the error propagation at each layer of the SIC receiver is eliminated by a genie.

In the first example, we simulate a relay system with $L=2$ hops and choose $N_{l}=5, l=1,2,3$, and $N_{b}=5$. The symbols are modulated by the 16-QAM constellations. Fig. 2 shows BERs of all systems versus $\mathrm{SNR}_{1}$ for $\mathrm{SNR}_{2}=20 \mathrm{~dB}$. It can be seen from Fig. 2 that the optimal DPC-based relay system has a better BER performance compared with the relay system using the SIC receiver, since the latter system suffers from error propagation.

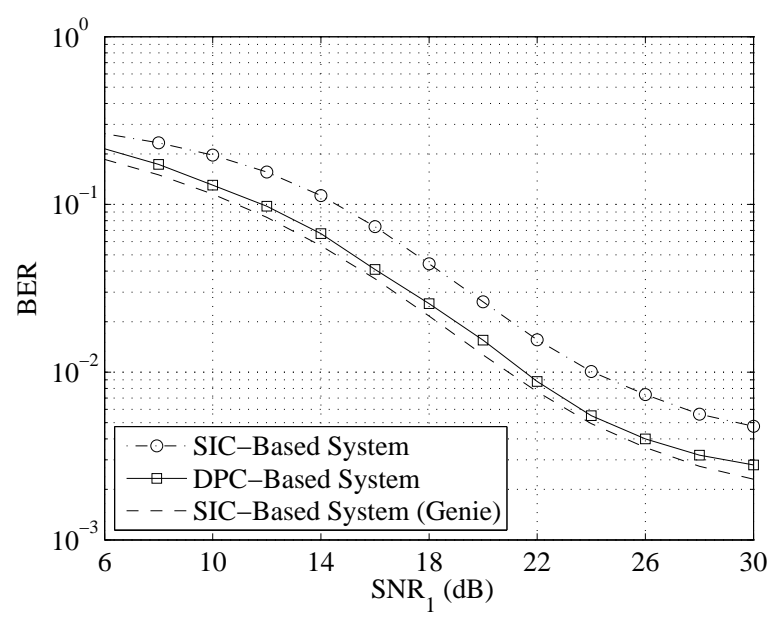

Fig. 2. Example 1: Two hops. $N_{l}=5, l=1,2,3, N_{b}=5$, 16-QAM, $\mathrm{SNR}_{2}=20 \mathrm{~dB}$

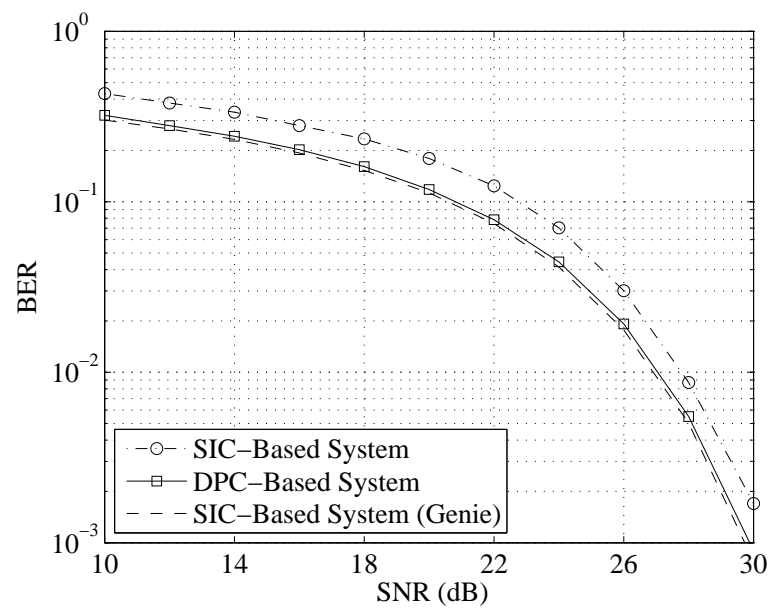

Fig. 3. Example 2: Five hops. $N_{l}=5, l=1, \cdots, 6, N_{b}=4$, 64-QAM, $\mathrm{SNR}_{l}=\mathrm{SNR}, l=1, \cdots, 5$.

In the second example, a multi-hop MIMO relay system with $L=5$ and $N_{l}=5, l=1, \cdots, 6$, and $N_{b}=4$ is simulated. Each hop has the same SNR, i.e., $\mathrm{SNR}_{l}=\mathrm{SNR}$, $l=1, \cdots, 5$. The 64-QAM constellations are used to modulate the symbols. Fig. 3 displays the BER performance of all three systems versus SNR. Obviously, for multi-hop systems, the DPC-based relay design outperforms the relay system using the SIC receiver by removing the error propagation effect. From Figs. 2 and 3, we observe a slight SNR loss of the DPC-based relay system compared with the genie-aided SICbased relay system. This is mainly due to the inherent power loss and modulo loss of the Tomlinson-Harashima precoder [16].

The reason for the error floor effect displayed in Fig. 2 for both the SIC-based system and the DPC-based system is that the relay system is fully-loaded in the sense that $N_{l}=N_{b}$, $l=1,2,3$. It is well-known that for a one-hop (point-to-point) MIMO system with an SIC receiver, the diversity order for the first decoded stream is only one if the transmitter and the receiver have the same number of antennas. Compared with 
that in Fig. 2, the system shown in Fig. 3 is under-loaded since $N_{l}>N_{b}, l=1, \cdots, 6$. Thus, the error floor effect is not observed in Fig. 3.

\section{CONCLUSIONS}

We have established the uplink-downlink duality of multihop AF-MIMO relay systems with any number of hops and any number of antennas at each node, which generalizes several previously established results. Based on such duality, we proposed an optimal design of the source precoding matrix and relay amplifying matrices for multi-hop AF-MIMO relay systems with a DPC-based transmitter at the source node. Simulation results show that the optimal DPC-based MIMO relay system has a lower BER than the optimal relay system using the SIC receiver.

\section{APPENDIX A}

\section{PROOF OF THEOREM 1}

The basic idea of the proof is to show under which conditions of $\mathbf{P}, \mathbf{Q}$, and $c_{l}, l=1, \cdots, L-1$, the uplink and downlink channels achieve identical SINRs. The proof is conducted in three steps. First, for both the uplink MIMO relay channel (6)-(8) and the downlink MIMO relay channel (3)-(5), we write the SINR for each data stream and the required total transmission power. Second, we rewrite the total transmission power of the downlink system based on the definition of duality that both channels should achieve identical SINRs. Finally, we find under which $\mathbf{P}, \mathbf{Q}$, and $c_{l}, l=1, \cdots, L-1$, the total transmission power consumed by both systems is identical.

Based on (7), the SINRs of data streams at the destination node of the uplink MIMO relay channel are given by (21) shown at the bottom of the next page. The transmission power $P_{l}^{U}$ consumed by the $l$ th node, $l=1, \cdots, L$, in the uplink relay system can be calculated using (6) and (7) as

$$
\begin{aligned}
P_{L+2-l}^{U}= & \mathbf{F}_{l-1}^{H} \mathrm{E}\left[\mathbf{y}_{L+2-l}^{U}\left(\mathbf{y}_{L+2-l}^{U}\right)^{H}\right] \mathbf{F}_{l-1} \\
= & \operatorname{tr}\left(\bigotimes_{m=l}^{L}\left(\mathbf{F}_{m-1}^{H} \mathbf{H}_{m}^{H}\right) \mathbf{V} \mathbf{P} \mathbf{V}^{H} \bigotimes_{m=L}^{l}\left(\mathbf{H}_{m} \mathbf{F}_{m-1}\right)\right. \\
& +\mathbf{F}_{l-1}^{H}\left(\sum_{k=l}^{L-1} \bigotimes_{m=l}^{k}\left(\mathbf{H}_{m}^{H} \mathbf{F}_{m}^{H}\right) \bigotimes_{m=k}^{l}\left(\mathbf{F}_{m} \mathbf{H}_{m}\right)\right. \\
& \left.\left.+\mathbf{I}_{N_{l}}\right) \mathbf{F}_{l-1}\right), \quad l=2, \cdots, L-1 \\
P_{2}^{U}= & \operatorname{tr}\left(\mathbf{F}{ }_{L-1}^{H}\left(\mathbf{H}_{L}^{H} \mathbf{V} \mathbf{P} \mathbf{V}^{H} \mathbf{H}_{L}+\mathbf{I}_{N_{L}}\right) \mathbf{F}_{L-1}\right) \\
P_{1}^{U}= & \operatorname{tr}(\mathbf{P}) .
\end{aligned}
$$

The total transmission power consumed by the uplink MIMO relay system can be obtained by the sum of (22)-(24) and written as

$$
\begin{gathered}
P_{T}^{U}=\sum_{i=1}^{N_{b}} p_{i}\left(1+\mathbf{v}_{i}^{H} \sum_{l=2}^{L}\left(\bigotimes_{m=L}^{l}\left(\mathbf{H}_{m} \mathbf{F}_{m-1}\right)\right.\right. \\
\left.\left.\bigotimes_{m=l}^{L}\left(\mathbf{F}_{m-1}^{H} \mathbf{H}_{m}^{H}\right)\right) \mathbf{v}_{i}\right)+\sum_{l=2}^{L-1} \operatorname{tr}\left(\mathbf { F } _ { l - 1 } ^ { H } \sum _ { k = l } ^ { L - 1 } \left(\bigotimes_{m=l}^{k}\left(\mathbf{H}_{m}^{H} \mathbf{F}_{m}^{H}\right)\right.\right. \\
\left.\left.\bigotimes_{m=k}^{l}\left(\mathbf{F}_{m} \mathbf{H}_{m}\right)\right) \mathbf{F}_{l-1}\right)+\sum_{l=1}^{L-1} \operatorname{tr}\left(\mathbf{F}_{l}^{H} \mathbf{F}_{l}\right) .
\end{gathered}
$$

Similarly, using (4), the SINRs of data streams at the destination node of the downlink relay channel are given by (26) shown at the bottom of the next page. The transmission power $P_{l}^{D}$ consumed by the $l$ th node, $l=1, \cdots, L$, in the downlink system can be obtained from (3) and (4) as

$$
\begin{aligned}
P_{l+1}^{D}= & \mathbf{F}_{l} \mathrm{E}\left[\mathbf{y}_{l+1}^{D}\left(\mathbf{y}_{l+1}^{D}\right)^{H}\right] \mathbf{F}_{l}^{H} \\
= & \operatorname{tr}\left(\bigotimes_{m=l}^{1}\left(c_{m} \mathbf{F}_{m} \mathbf{H}_{m}\right) \mathbf{U} \mathbf{Q} \mathbf{U}^{H} \bigotimes_{m=1}^{l}\left(c_{m} \mathbf{H}_{m}^{H} \mathbf{F}_{m}^{H}\right)+c_{l}^{2}\right. \\
& \times \mathbf{F}_{l}\left(\sum_{k=2}^{l} \bigotimes_{m=l}^{k}\left(c_{m-1} \mathbf{H}_{m} \mathbf{F}_{m-1}\right) \bigotimes_{m=k}^{l}\left(c_{m-1} \mathbf{F}_{m-1}^{H} \mathbf{H}_{m}^{H}\right)\right. \\
& \left.\left.+\mathbf{I}_{N_{l+1}}\right) \mathbf{F}_{l}^{H}\right), \quad l=2, \cdots, L-1 \\
P_{2}^{D}= & \operatorname{tr}\left(c_{1}^{2} \mathbf{F}_{1}\left(\mathbf{H}_{1} \mathbf{U} \mathbf{Q} \mathbf{U}^{H} \mathbf{H}_{1}^{H}+\mathbf{I}_{N_{2}}\right) \mathbf{F}_{1}^{H}\right) \\
P_{1}^{D}= & \operatorname{tr}(\mathbf{Q}) .
\end{aligned}
$$

The total transmission power consumed by the downlink MIMO relay system is obtained by the sum of (27)-(29) and given by

$$
\begin{aligned}
P_{T}^{D}= & \sum_{i=1}^{N_{b}} q_{i}\left(1+\mathbf{u}_{i}^{H} \sum_{l=1}^{L-1}\left(\bigotimes_{m=1}^{l}\left(c_{m} \mathbf{H}_{m}^{H} \mathbf{F}_{m}^{H}\right) \bigotimes_{m=l}^{1}\left(c_{m} \mathbf{F}_{m} \mathbf{H}_{m}\right)\right)\right. \\
& \left.\times \mathbf{u}_{i}\right)+\sum_{l=2}^{L-1} \operatorname{tr}\left(c _ { l } ^ { 2 } \mathbf { F } _ { l } \sum _ { k = 2 } ^ { l } \left(\bigotimes_{m=l}^{k}\left(c_{m-1} \mathbf{H}_{m} \mathbf{F}_{m-1}\right)\right.\right. \\
& \left.\left.\bigotimes_{m=k}^{l}\left(c_{m-1} \mathbf{F}_{m-1}^{H} \mathbf{H}_{m}^{H}\right)\right) \mathbf{F}_{l}^{H}\right)+\sum_{l=1}^{L-1} \operatorname{tr}\left(c_{l}^{2} \mathbf{F}_{l} \mathbf{F}_{l}^{H}\right) .
\end{aligned}
$$

To achieve identical SINRs at the uplink and the downlink systems, we should have $\operatorname{SINR}_{i}^{U}=\operatorname{SINR}_{i}^{D}, i=1, \cdots, N_{b}$. Please note that we do not assume that all data streams have identical SINR, i.e., it is possible that $\operatorname{SINR}_{i}^{U} \neq \operatorname{SINR}_{j}^{U}$, for $i \neq j$. Using (21) and (26) we obtain from $\sum_{i=1}^{N_{b}} \operatorname{SINR}_{i}^{U}=$ $\sum_{i=1}^{N_{b}} \operatorname{SINR}_{i}^{D}$ that

$$
\begin{aligned}
& \sum_{i=1}^{N_{b}}\left(\prod _ { l = 1 } ^ { L - 1 } c _ { l } ^ { 2 } q _ { i } \left(\sum_{\substack{j=1 \\
j \neq i}}^{N_{b}}\left|\mathbf{u}_{i}^{H} \bigotimes_{l=1}^{L-1}\left(\mathbf{H}_{l}^{H} \mathbf{F}_{l}^{H}\right) \mathbf{H}_{L}^{H} \mathbf{v}_{j}\right|^{2} p_{j}\right.\right. \\
& \left.\left.+\mathbf{u}_{i}^{H} \sum_{l=1}^{L-1}\left(\bigotimes_{m=1}^{l}\left(\mathbf{H}_{m}^{H} \mathbf{F}_{m}^{H}\right) \bigotimes_{m=l}^{1}\left(\mathbf{F}_{m} \mathbf{H}_{m}\right)\right) \mathbf{u}_{i}+1\right)\right)= \\
& \sum_{i=1}^{N_{b}} p_{i}\left(\sum_{\substack{j=1 \\
j \neq i}}^{N_{b}}\left|\mathbf{v}_{i}^{H} \mathbf{H}_{L} \bigotimes_{l=L-1}^{1}\left(c_{l} \mathbf{F}_{l} \mathbf{H}_{l}\right) \mathbf{u}_{j}\right|^{2} q_{j}+\mathbf{v}_{i}^{H}\right. \\
& \left.\times \sum_{l=2}^{L}\left(\bigotimes_{\substack{l \\
m=L}}^{L}\left(c_{m-1} \mathbf{H}_{m} \mathbf{F}_{m-1}\right) \bigotimes_{m=l}^{\bigotimes}\left(c_{m-1} \mathbf{F}_{m-1}^{H} \mathbf{H}_{m}^{H}\right)\right) \mathbf{v}_{i}+1\right) .(31
\end{aligned}
$$

By using the identity of

$$
\begin{aligned}
\sum_{i=1}^{N_{b}} \sum_{j=1, j \neq i}^{N_{b}} q_{i}\left|\mathbf{u}_{i}^{H} \bigotimes_{l=1}^{L-1}\left(c_{l} \mathbf{H}_{l}^{H} \mathbf{F}_{l}^{H}\right) \mathbf{H}_{L}^{H} \mathbf{v}_{j}\right|^{2} p_{j} \\
=\sum_{i=1}^{N_{b}} \sum_{j=1, j \neq i}^{N_{b}} p_{i}\left|\mathbf{v}_{i}^{H} \mathbf{H}_{L} \bigotimes_{l=L-1}^{1}\left(c_{l} \mathbf{F}_{l} \mathbf{H}_{l}\right) \mathbf{u}_{j}\right|^{2} q_{j}
\end{aligned}
$$


we obtain from (31) that

Substituting (32) back into (30), we can rewrite $P_{T}^{D}$ as

$$
\begin{aligned}
P_{T}^{D}= & \operatorname{tr}\left(\sum_{l=2}^{L} \prod_{m=l}^{L} c_{m-1}^{2} \bigotimes_{m=l}^{L}\left(\mathbf{F}_{m-1}^{H} \mathbf{H}_{m}^{H}\right) \mathbf{V} \mathbf{P} \mathbf{V}^{H}\right. \\
& \left.\bigotimes_{m=L}^{l}\left(\mathbf{H}_{m} \mathbf{F}_{m-1}\right)+\mathbf{P}\right)+\operatorname{tr}\left(\sum_{l=1}^{L-2}\left(1-\prod_{m=l+1}^{L-1} c_{m}^{2}\right)\right. \\
& \bigotimes_{m=1}^{l}\left(c_{m} \mathbf{H}_{m}^{H} \mathbf{F}_{m}^{H}\right) \bigotimes_{m=l}\left(c_{m} \mathbf{F}_{m} \mathbf{H}_{m}\right) \mathbf{U} \mathbf{Q} \mathbf{U}^{H} \\
& \left.+\left(1-\prod_{m=1}^{L-1} c_{m}^{2}\right) \mathbf{U} \mathbf{Q} \mathbf{U}^{H}\right) \\
& +\sum_{l=2}^{L-1} \operatorname{tr}\left(c _ { l } ^ { 2 } \mathbf { F } _ { l } \sum _ { k = 2 } ^ { l } \left(\bigotimes_{m=l}^{k}\left(c_{m-1} \mathbf{H}_{m} \mathbf{F}_{m-1}\right)\right.\right. \\
& \left.\left.\bigotimes_{l}^{l}\left(c_{m-1} \mathbf{F}_{m-1}^{H} \mathbf{H}_{m}^{H}\right)\right) \mathbf{F}_{l}^{H}\right)+\sum_{l=1}^{L-1} \operatorname{tr}\left(c_{l}^{2} \mathbf{F}_{l} \mathbf{F}_{l}^{H}\right) .(33)
\end{aligned}
$$

For notational simplicity, let us denote

$$
\begin{aligned}
a_{l} \triangleq & \operatorname{tr}\left(c _ { l } ^ { 2 } \mathbf { F } _ { l } \sum _ { k = 2 } ^ { l } \left(\bigotimes_{m=l}^{k}\left(c_{m-1} \mathbf{H}_{m} \mathbf{F}_{m-1}\right)\right.\right. \\
& \left.\left.\bigotimes_{m=k}^{l}\left(c_{m-1} \mathbf{F}_{m-1}^{H} \mathbf{H}_{m}^{H}\right)\right) \mathbf{F}_{l}^{H}\right), \quad l=2, \cdots, L-1 .
\end{aligned}
$$

Then with some manipulations, we have

$$
\begin{aligned}
\sum_{l=2}^{L-1} a_{l} & =\sum_{l=2}^{L-1} \prod_{m=l+1}^{L-1} c_{m}^{2} a_{l}+\sum_{l=2}^{L-1}\left(1-\prod_{m=l+1}^{L-1} c_{m}^{2}\right) a_{l} \\
& =\sum_{l=2}^{L-1} \sum_{k=l}^{L-1} \prod_{m=k+1}^{L-1} c_{m}^{2} \operatorname{tr}\left(c_{k}^{2} \mathbf{F}_{k} \bigotimes_{m=k}^{l}\left(c_{m-1} \mathbf{H}_{m} \mathbf{F}_{m-1}\right)\right.
\end{aligned}
$$

$$
\begin{gathered}
\prod_{l=1}^{L-1} c_{l}^{2} \sum_{i=1}^{N_{b}} q_{i}\left(\mathbf{u}_{i}^{H} \sum_{l=1}^{L-1}\left(\bigotimes_{m=1}^{l}\left(\mathbf{H}_{m}^{H} \mathbf{F}_{m}^{H}\right) \bigotimes_{m=l}^{1}\left(\mathbf{F}_{m} \mathbf{H}_{m}\right)\right) \mathbf{u}_{i}+1\right) \\
=\sum_{i=1}^{N_{b}} p_{i}\left(\mathbf { v } _ { i } ^ { H } \sum _ { l = 2 } ^ { L } \left(\bigotimes_{m=L}^{l}\left(c_{m-1} \mathbf{H}_{m} \mathbf{F}_{m-1}\right)\right.\right. \\
\left.\left.\bigotimes_{m=l}^{L}\left(c_{m-1} \mathbf{F}_{m-1}^{H} \mathbf{H}_{m}^{H}\right)\right) \mathbf{v}_{i}+1\right) .
\end{gathered}
$$

$$
\begin{aligned}
& \left.\bigotimes_{m=l}^{k}\left(c_{m-1} \mathbf{F}_{m-1}^{H} \mathbf{H}_{m}^{H}\right) \mathbf{F}_{k}^{H}\right)+\sum_{l=2}^{L-1}\left(1-\prod_{m=l+1}^{L-1} c_{m}^{2}\right) a_{l} \\
= & \sum_{l=2}^{L-1} \sum_{k=l}^{L-1} \prod_{m=k+1}^{L-1} c_{m}^{2} \operatorname{tr}\left(c_{l-1}^{2} \mathbf{F}_{l-1}^{H} \bigotimes_{m=l}^{k}\left(c_{m} \mathbf{H}_{m}^{H} \mathbf{F}_{m}^{H}\right)\right. \\
& \left.\bigotimes_{m=k}^{l}\left(c_{m} \mathbf{F}_{m} \mathbf{H}_{m}\right) \mathbf{F}_{l-1}\right)+\sum_{l=2}^{L-1}\left(1-\prod_{m=l+1}^{L-1} c_{m}^{2}\right) a_{l} \\
= & \sum_{l=2}^{L-1} \prod_{m=l}^{L} c_{m-1}^{2} \sum_{k=l}^{L-1} \operatorname{tr}\left(\mathbf{F}_{l-1}^{H} \bigotimes_{m=l}^{k}\left(\mathbf{H}_{m}^{H} \mathbf{F}_{m}^{H}\right)\right. \\
& \left.\bigotimes_{m=k}^{l}\left(\mathbf{F}_{m} \mathbf{H}_{m}\right) \mathbf{F}_{l-1}\right)+\sum_{l=2}^{L-1}\left(1-\prod_{m=l+1}^{L-1} c_{m}^{2}\right) a_{l}
\end{aligned}
$$

and

$$
\begin{aligned}
& \sum_{l=1}^{L-1} \operatorname{tr}\left(c_{l}^{2} \mathbf{F}_{l} \mathbf{F}_{l}^{H}\right)=\sum_{l=2}^{L} \prod_{m=l}^{L} c_{m-1}^{2} \operatorname{tr}\left(\mathbf{F}_{l-1}^{H} \mathbf{F}_{l-1}\right) \\
& +\sum_{l=1}^{L-1}\left(1-\prod_{m=l+1}^{L-1} c_{m}^{2}\right) \operatorname{tr}\left(c_{l}^{2} \mathbf{F}_{l} \mathbf{F}_{l}^{H}\right)
\end{aligned}
$$

Substituting (35) and (36) back into (33) and after rearranging terms, we can rewrite $P_{T}^{D}$ as

$$
\begin{aligned}
& P_{T}^{D}=\sum_{l=2}^{L-1} \prod_{m=l}^{L} c_{m-1}^{2} \operatorname{tr}\left(\bigotimes_{m=l}^{L}\left(\mathbf{F}_{m-1}^{H} \mathbf{H}_{m}^{H}\right) \mathbf{V P V} \mathbf{V}_{m=L}^{H}\left(\mathbf{H}_{m} \mathbf{F}_{m-1}\right)\right. \\
& \left.+\mathbf{F}_{l-1}^{H}\left(\sum_{k=l}^{L-1} \bigotimes_{m=l}^{k}\left(\mathbf{H}_{m}^{H} \mathbf{F}_{m}^{H}\right) \bigotimes_{m=k}^{l}\left(\mathbf{F}_{m} \mathbf{H}_{m}\right)+\mathbf{I}_{N_{l}}\right) \mathbf{F}_{l-1}\right) \\
& +c_{L-1}^{2} \operatorname{tr}\left(\mathbf{F}_{L-1}^{H}\left(\mathbf{H}_{L}^{H} \mathbf{V} \mathbf{P} \mathbf{V}^{H} \mathbf{H}_{L}+\mathbf{I}_{N_{L}}\right) \mathbf{F}_{L-1}\right)+\operatorname{tr}(\mathbf{P}) \\
& +\sum_{l=2}^{L-2}\left(1-\prod_{m=l+1}^{L-1} c_{m}^{2}\right) \operatorname{tr}\left(\bigotimes_{m=1}^{l}\left(c_{m} \mathbf{H}_{m}^{H} \mathbf{F}_{m}^{H}\right) \bigotimes_{m=l}^{1}\left(c_{m} \mathbf{F}_{m} \mathbf{H}_{m}\right)\right. \\
& \times \mathbf{U Q U}^{H}+c_{l}^{2} \mathbf{F}_{l}\left(\sum_{k=2}^{l} \bigotimes_{m=l}^{k}\left(c_{m-1} \mathbf{H}_{m} \mathbf{F}_{m-1}\right)\right. \\
& \left.\left.\bigotimes_{m=k}^{l}\left(c_{m-1} \mathbf{F}_{m-1}^{H} \mathbf{H}_{m}^{H}\right)+\mathbf{I}_{N_{l+1}}\right) \mathbf{F}_{l}^{H}\right) \\
& +\left(1-\prod_{m=2}^{L-1} c_{m}^{2}\right) \operatorname{tr}\left(c_{1}^{2} \mathbf{F}_{1}\left(\mathbf{H}_{1} \mathbf{U} \mathbf{Q} \mathbf{U}^{H} \mathbf{H}_{1}^{H}+\mathbf{I}_{N_{2}}\right) \mathbf{F}_{1}^{H}\right) \\
& +\left(1-\prod_{m=1}^{L-1} c_{m}^{2}\right) \operatorname{tr}\left(\mathbf{U Q U}^{H}\right) \text {. }
\end{aligned}
$$

$$
\operatorname{SINR}_{i}^{U}=\frac{\left|\mathbf{u}_{i}^{H} \bigotimes_{l=1}^{L-1}\left(\mathbf{H}_{l}^{H} \mathbf{F}_{l}^{H}\right) \mathbf{H}_{L}^{H} \mathbf{v}_{i}\right|^{2} p_{i}}{\sum_{\substack{j=1 \\ j \neq i}}^{N_{b}}\left|\mathbf{u}_{i}^{H} \bigotimes_{l=1}^{L-1}\left(\mathbf{H}_{l}^{H} \mathbf{F}_{l}^{H}\right) \mathbf{H}_{L}^{H} \mathbf{v}_{j}\right|^{2} p_{j}+\mathbf{u}_{i}^{H} \sum_{l=1}^{L-1}\left(\bigotimes_{m=1}^{l}\left(\mathbf{H}_{m}^{H} \mathbf{F}_{m}^{H}\right) \bigotimes_{m=l}^{1}\left(\mathbf{F}_{m} \mathbf{H}_{m}\right)\right) \mathbf{u}_{i}+1}, \quad i=1, \cdots, N_{b} .
$$$$
\operatorname{SINR}_{i}^{D}=\frac{\left|\mathbf{v}_{i}^{H} \mathbf{H}_{L} \bigotimes_{l=L-1}^{1}\left(c_{l} \mathbf{F}_{l} \mathbf{H}_{l}\right) \mathbf{u}_{i}\right|^{2} q_{i}}{\sum_{\substack{j=1 \\ j \neq i}}^{N_{b}}\left|\mathbf{v}_{i}^{H} \mathbf{H}_{L} \bigotimes_{l=L-1}^{1}\left(c_{l} \mathbf{F}_{l} \mathbf{H}_{l}\right) \mathbf{u}_{j}\right|^{2} q_{j}+\mathbf{v}_{i}^{H} \sum_{l=2}^{L}\left(\bigotimes_{m=L}^{l}\left(c_{m-1} \mathbf{H}_{m} \mathbf{F}_{m-1}\right) \bigotimes_{m=l}^{L}\left(c_{m-1} \mathbf{F}_{m-1}^{H} \mathbf{H}_{m}^{H}\right)\right) \mathbf{v}_{i}+1}, i=1, \cdots, N_{b} .
$$ 
Using the expressions of $P_{l}^{U}$ in (22)-(24) and $P_{l}^{D}$ in (27)-(29), $l=1, \cdots, L,(37)$ can be rewritten as

$$
\begin{aligned}
P_{T}^{D}= & \sum_{l=2}^{L-1} \prod_{m=l}^{L} c_{m-1}^{2} P_{L+2-l}^{U}+c_{L-1}^{2} P_{2}^{U}+P_{1}^{U} \\
& +\sum_{l=2}^{L-2}\left(1-\prod_{m=l+1}^{L-1} c_{m}^{2}\right) P_{l+1}^{D}+\sum_{l=1}^{2}\left(1-\prod_{m=l}^{L-1} c_{m}^{2}\right) P_{l}^{D} \\
= & \sum_{l=1}^{L-1} \prod_{m=l}^{L-1} c_{m}^{2} P_{L+1-l}^{U}+P_{1}^{U}+\sum_{l=1}^{L-1}\left(1-\prod_{m=l}^{L-1} c_{m}^{2}\right) P_{l}^{D} .
\end{aligned}
$$

Since the uplink and downlink systems should consume the same amount of total transmission power, we have

$$
P_{T}^{D}-P_{T}^{U}=\sum_{l=1}^{L-1}\left(\prod_{m=l}^{L-1} c_{m}^{2}-1\right)\left(P_{L+1-l}^{U}-P_{l}^{D}\right)=0 .
$$

Obviously, for any $L \geq 2$, (38) is true if $\prod_{m=l}^{L-1} c_{m}^{2}=1$ for $l=$ $1, \cdots, L-1$, which is equivalent to $c_{l}=1, l=1, \cdots, L-1$. Thus, the first part of Theorem 1 (without transmission power constraint at each node) is proven. Moreover, (38) also holds if $P_{L+1-l}^{U}=P_{l}^{D}, l=1, \cdots, L-1$. Then we have $P_{1}^{U}=$ $P_{L}^{D}$ due to $P_{T}^{D}=P_{T}^{U}$. With transmission power constraint at individual nodes, there is $P_{l}^{D} \leq \rho_{l}^{D}$ and $P_{l}^{U} \leq \rho_{l}^{U}, l=$ $1, \cdots, L$. Obviously, to optimize the system performance, all available power should be exploited, i.e., $P_{l}^{D}=\rho_{l}^{D}$ and $P_{l}^{U}=$ $\rho_{l}^{U}, l=1, \cdots, L$. Thus, we have $\rho_{L+1-l}^{U}=\rho_{l}^{D}, l=1, \cdots, L$, and the second part of Theorem 1 (with transmission power constraint at individual nodes) is proven.

\section{APPENDIX B}

\section{PROOF OF THEOREM 2}

With an SIC receiver, the source symbols are detected successively with the last symbol detected first and the first symbol detected last, and the interference from detected symbols is subtracted. Therefore, the SINRs of data streams at the destination node can be written as (39) shown at the bottom of this page. For a MIMO relay system employing a DPC transmitter at the source node, the information-bearing symbols are encoded successively with the first symbol encoded first and the last symbol encoded last, and the interference from encoded symbols is removed. Thus, the SINRs of data streams at the destination node of the DPC-based downlink
MIMO relay channel is given by (40) shown at the bottom of this page. Using (39) and (40), and the identity of

$$
\begin{aligned}
\sum_{i=1}^{N_{b}} & \sum_{j=1}^{i-1} q_{i}\left|\mathbf{u}_{i}^{H} \bigotimes_{l=1}^{L-1}\left(c_{l} \mathbf{H}_{l}^{H} \mathbf{F}_{l}^{H}\right) \mathbf{H}_{L}^{H} \mathbf{v}_{j}\right|^{2} p_{j} \\
= & \sum_{i=1}^{N_{b}} \sum_{j=i+1}^{N_{b}} p_{i}\left|\mathbf{v}_{i}^{H} \mathbf{H}_{L} \bigotimes_{l=L-1}^{1}\left(c_{l} \mathbf{F}_{l} \mathbf{H}_{l}\right) \mathbf{u}_{j}\right|^{2} q_{j}
\end{aligned}
$$

we obtain from $\sum_{i=1}^{N_{b}} \operatorname{SINR}_{i}^{U}=\sum_{i=1}^{N_{b}} \operatorname{SINR}_{i}^{D}$ the expression of $P_{T}^{D}$ as in (33), and the steps in (34)-(38) remain valid. Thus Theorem 2 is proven.

\section{REFERENCES}

[1] B. Wang, J. Zhang, and A. Høst-Madsen, "On the capacity of MIMO relay channels," IEEE Trans. Inf. Theory, vol. 51, pp. 29-43, Jan. 2005.

[2] X. Tang and Y. Hua, "Optimal design of non-regenerative MIMO wireless relays," IEEE Trans. Wireless Commun., vol. 6, pp. 1398-1407, Apr. 2007.

[3] Y. Rong, X. Tang, and Y. Hua, "A unified framework for optimizing linear non-regenerative multicarrier MIMO relay communication systems,' IEEE Trans. Signal Process., vol. 57, pp. 4837-4851, Dec. 2009.

[4] Y. Rong, "Optimal linear non-regenerative multi-hop MIMO relays with MMSE-DFE receiver at the destination," IEEE Trans. Wireless Commun., vol. 9, pp. 2268-2279, Jul. 2010.

[5] D. Tse and P. Viswanath, Fundamentals of Wireless Communication. Cambridge University Press, 2005.

[6] K. S. Gomadam and S. A. Jafar, "Duality of MIMO multiple access channel and broadcast channel with amplify-and-forward relays," IEEE Trans. Commun., vol. 58, pp. 211-217, Jan. 2010.

[7] P. Viswanath and D. N. C. Tse, "Sum capacity of the vector Gaussian broadcast channel and uplink-downlink duality," IEEE Trans. Inf. Theory, vol. 49, pp. 1912-1921, Aug. 2003.

[8] N. Jindal, S. Vishwanath, and A. Goldsmith, "On the duality of Gaussian multiple-access and broadcast channels," IEEE Trans. Inf. Theory, vol. 50, pp. 768-783, May 2004.

[9] S. A. Jafar, K. S. Gomadam, and C. Huang, "Duality and rate optimization for multiple access and broadcast channels with amplifyand-forward relays," IEEE Trans. Inf. Theory, vol. 53, pp. 3350-3370, Oct. 2007.

[10] D. P. Palomar and Y. Jiang, MIMO Transceiver Design via Majorization Theory. Now Publishers, 2007.

[11] M. H. M. Costa, "Writing on dirty paper," IEEE Trans. Inf. Theory, vol. 29, pp. 439-441, May 1983.

[12] Y. Rong and Y. Hua, "Optimality of diagonalization of multi-hop MIMO relays," IEEE Trans. Wireless Commun., vol. 8, pp. 6068-6077, Dec. 2009.

[13] A. W. Marshall and I. Olkin, Inequalities: Theory of Majorization and Its Applications. Academic Press, 1979.

[14] M. Tomlinson, "New automatic equaliser employing modulo arithmetic," Electronics Lett., vol. 7, pp. 138-139, Mar. 1971.

[15] H. Harashima and H. Miyakawa, "Matched-transmission technique for channels with intersymbol interference," IEEE Trans. Commun., vol. 20, pp. 774-780, Aug. 1972.

$$
\begin{gathered}
\operatorname{SINR}_{i}^{U}=\frac{\left|\mathbf{u}_{i}^{H} \bigotimes_{l=1}^{L-1}\left(\mathbf{H}_{l}^{H} \mathbf{F}_{l}^{H}\right) \mathbf{H}_{L}^{H} \mathbf{v}_{i}\right|^{2} p_{i}}{\sum_{j=1}^{i-1}\left|\mathbf{u}_{i}^{H} \bigotimes_{l=1}^{L-1}\left(\mathbf{H}_{l}^{H} \mathbf{F}_{l}^{H}\right) \mathbf{H}_{L}^{H} \mathbf{v}_{j}\right|^{2} p_{j}+\mathbf{u}_{i}^{H} \sum_{l=1}^{L-1}\left(\bigotimes_{m=1}^{l}\left(\mathbf{H}_{m}^{H} \mathbf{F}_{m}^{H}\right) \bigotimes_{m=l}^{1}\left(\mathbf{F}_{m} \mathbf{H}_{m}\right)\right) \mathbf{u}_{i}+1}, \quad i=1, \cdots, N_{b} . \\
\operatorname{SINR}_{i}^{D}=\frac{\left|\mathbf{v}_{i}^{H} \mathbf{H}_{L} \bigotimes_{l=L-1}^{1}\left(c_{l} \mathbf{F}_{l} \mathbf{H}_{l}\right) \mathbf{u}_{i}\right|^{2} q_{i}}{\sum_{j=i+1}^{N_{b}}\left|\mathbf{v}_{i}^{H} \mathbf{H}_{L} \bigotimes_{l=L-1}^{\infty}\left(c_{l} \mathbf{F}_{l} \mathbf{H}_{l}\right) \mathbf{u}_{j}\right|^{2} q_{j}+\mathbf{v}_{i}^{H} \sum_{l=2}^{L}\left(\bigotimes_{m=L}^{l}\left(c_{m-1} \mathbf{H}_{m} \mathbf{F}_{m-1}\right) \bigotimes_{m=l}^{L}\left(c_{m-1} \mathbf{F}_{m-1}^{H} \mathbf{H}_{m}^{H}\right)\right) \mathbf{v}_{i}+1}, i=1, \cdots, N_{b} .
\end{gathered}
$$


[16] S. Shamai and L. Laroia, "The inter-symbol interference channel: Lower bounds on capacity and channel precoding loss," IEEE Trans. Inf. Theory, vol. 42, pp. 1388-1404, Sep. 1996.

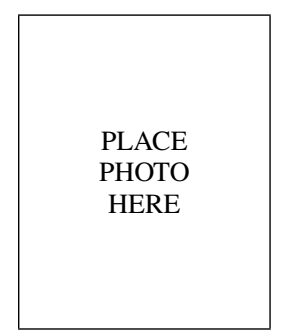

Yue Rong (S'03-M'06-SM'11) received the B.E degree from Shanghai Jiao Tong University, China, the M.Sc. degree from the University of DuisburgEssen, Duisburg, Germany, and the Ph.D. degree (summa cum laude) from Darmstadt University of Technology, Darmstadt, Germany, all in electrical engineering, in 1999, 2002, and 2005, respectively.

From April 2001 to October 2001, he was a research assistant at the Fraunhofer Institute of Microelectronic Circuits and Systems, Duisburg, Germany. From October 2001 to March 2002, he was with Nokia Ltd., Bochum, Germany. From November 2002 to March 2005, he was a Research Associate at the Department of Communication Systems in the University of Duisburg-Essen. From April 2005 to January 2006, he was with the Institute of Telecommunications at Darmstadt University of Technology, as a Research Associate. From February 2006 to November 2007, he was a Postdoctoral Researcher with the Department of Electrical Engineering, University of California, Riverside. Since December 2007, he has been with the Department of Electrical and Computer Engineering, Curtin University of Technology, Perth, Australia, where he is now a Senior Lecturer. His research interests include signal processing for communications, wireless communications, wireless networks, applications of linear algebra and optimization methods, and statistical and array signal processing.

Dr. Rong received the Best Paper Award at the 16th Asia-Pacific Conference on Communications, Auckland, New Zealand, 2010, the 2010 Young Researcher of the Year Award of the Faculty of Science and Engineering at Curtin University, the 2004 Chinese Government Award for Outstanding SelfFinanced Students Abroad (China), and the 2001-2002 DAAD/ABB Graduate Sponsoring Asia Fellowship (Germany). He has co-authored more than 50 referred IEEE journal and conference papers. He is a Guest Editor of the IEEE JSAC special issue on Theories and Methods for Advanced Wireless Relays to be published in 2012, a Guest Editor of the EURASIP JASP Special Issue on Signal Processing Methods for Diversity and Its Applications to be published in 2012, and has served as a TPC member for IEEE ICC, IWCMC, and ChinaCom.

\begin{tabular}{|c|}
\hline \\
PLACE \\
PHOTO \\
HERE \\
\end{tabular}

Muhammad R. A. Khandaker (S'10) received the Bachelor of Science (Honours) degree in Computer Science and Engineering from Jahangirnagar University, Dhaka, Bangladesh in 2006 and Master of Science in Telecommunications Engineering from East West University, Dhaka, Bangladesh in 2007. He is currently working toward the Ph.D. degree at the Department of Electrical and Computer Engineering, Curtin University, Australia.

Mr. Khandaker started his career as a Jr. Hardware Design Engineer in Visual Magic Corporation Limited (VMCL-BD) in November 2005. He joined as a Lecturer in the Department of Computer Science and Engineering, IBAIS University, Dhaka, Bangladesh in January 2006. In November 2007, he joined as a Lecturer in the Department of Information and Communication Technology of Mawlana Bhasani Science and Technology University, Bangladesh. In January 2008, he joined as a Lecturer in the Institute of Information Technology (former Computer and Information Technology Institute), Jahangirnagar University, Dhaka, Bangladesh.

He was awarded Curtin International Postgraduate Research Scholarship (CIPRS) for his Ph.D. study in 2009. He also received the Best Paper Award at the 16th Asia-Pacific Conference on Communications, Auckland, New Zealand, 2010. 